\title{
DETERMINATION OF COVERAGE INTERVAL OF MICROPROTEIN IN URINE IN PREGNANT LADIES BY THE PYROGALLOL-RED METHOD IN PATIENTS ATTENDING A TERTIARY CARE HOSPITAL IN EASTERN INDIA
}

\author{
Purvita Dam ${ }^{1}$, Sharmistha Chatterjee 2 , Sanjoy Kunti3 ${ }^{3}$, Indranil Chakraborty4, Sanghamitra Chakraborty5 \\ ${ }^{1}$ Associate Professor, Department of Gynaecology and Obstetrics, College of Medicine and Sagore Dutta Hospital, Kolkata. \\ ${ }^{2}$ Assistant Professor, Department of Biochemistry, College of Medicine and Sagore Dutta Hospital, Kolkata. \\ ${ }_{3}^{3}$ Assistant Professor, Department of Biochemistry, College of Medicine and Sagore Dutta Hospital, Kolkata. \\ 4 Professor and HOD, Department of Biochemistry, College of Medicine and Sagore Dutta Hospital, Kolkata. \\ 5 Demonstrator, Department of Biochemistry, N.R.S. Medical College and Hospital, Kolkata.
}

\begin{abstract}
\section{BACKGROUND}

Pre-eclampsia characterised by hypertension and proteinuria in pregnancy is a major cause of maternal morbidity worldwide. So it is important to quantify the protein excretion in urine during pregnancy to help clinicians to predict pre-eclampsia in vulnerable patients. Traditionally, the estimation of 24 hours protein excretion in urine has been used for this purpose which is a cumbersome process. The dipstick method, though an easier one, does not yield satisfactory results. The pyrogallol-red method is perhaps a more acceptable method in this regard. It has also been noted that there exists almost no relevant data on pregnancy proteinuria in this part of the country. In light of this background, we embarked on this study to determine the coverage interval of microprotein in urine by the pyrogallol-red method in pregnant patients attending a tertiary care hospital in Eastern India.
\end{abstract}

\section{MATERIALS AND METHODS}

This cross-sectional study was carried out on 71 pregnant ladies attending the antenatal clinic in the Gynaecology and Obstetrics Department at College of Medicine and Sagore Dutta Hospital, a tertiary care hospital in Kolkata. The protein in fresh, clean voided urine of pregnant patients was estimated by the pyrogallol-red method on ERBA Chem $5 \mathrm{x}$ semi-auto analyser. The statistical data thus obtained was analysed by IBM SPSS version 16.

\section{RESULTS}

It may not be always feasible to collect 120 samples to establish a reference interval as required by the IFCC. For this reason, the term coverage interval is preferred over reference interval by IUPAC. The coverage interval of pregnancy proteinuria was calculated to be $23.99-29.64 \mathrm{mg} / \mathrm{dL}$ with a confidence of 0.90 and uncertainty of 0.049 .

\section{CONCLUSION}

In this study, we conclude that the coverage interval of urinary microprotein for pregnancy proteinuria is $23.99-29.64 \mathrm{mg} / \mathrm{dL}$ as determined by the pyrogallol-red method.

\section{KEYWORDS}

Coverage Interval, Pregnancy Proteinuria, Pyrogallol-red Method, Uncertainty.

HOW TO CITE THIS ARTICLE: Dam P, Chatterjee S, Kunti S, et al. Determination of coverage interval of microprotein in urine in pregnant ladies by the pyrogallol-red method in patients attending a tertiary care hospital in Eastern India. J. Evolution Med. Dent. Sci. 2017;6(91):6395-6398, DOI: 10.14260/jemds/2017/1390

\section{BACKGROUND}

Pregnancy pre-eclampsia is a major cause of maternal morbidity complicating up to $2-8 \%$ of pregnancies worldwide.[1,2] It is a multisystem disorder characterised by hypertension and proteinuria occurring after 20 weeks of pregnancy in previously normotensive women. It is important for clinicians caring for pregnant women to attempt to quantify proteinuria in pregnancy, with the aim of making a diagnosis as well as to anticipate the foetal and maternal outcome. Usually, any protein excretion greater than $150 \mathrm{mg}$

'Financial or Other Competing Interest': None.

Submission 11-10-2017, Peer Review 08-11-2017,

Acceptance 15-11-2017, Published 27-11-2017.

Corresponding Author:

Dr. Sharmistha Chatterjee,

Assistant Professor,

Department of Biochemistry,

College of Medicine and Sagore Dutta Hospital,

Kolkata.

E-mail: sharmisthacmajumder@yahoo.co.in

DOI: $10.14260 /$ jemds $/ 2017 / 1390$ daily is considered to be abnormal.[3] But in pregnant women, the protein excretion increases to about $300 \mathrm{mg}$ per day due to physiological changes.[4]

Traditionally, the gold standard for quantification of proteinuria has been the estimation of 24 hours excretion of protein in urine which has a cumbersome process of collection and is often inconvenient for ambulatory patients. [5] The dipstick method requires a spot urine sample and is a rapid, user-friendly, inexpensive and widely used method for estimation of proteinuria with the purpose of detection of pre-eclampsia during antenatal visits.[6] But studies have shown poor accuracy, low sensitivity and specificity associated with the dipstick method.[7-9]

There is a paucity of data related to the coverage interval of microprotein in urine in pregnant ladies in this part of the country. In this study, we attempted to determine the coverage interval of microprotein in urine of pregnant ladies attending the antenatal outdoor in this hospital by the pyrogallol-red method. We embarked on this study with the idea that this method of estimation of the microprotein may be used as a screening method for detection of proteinuria in 
pregnancy and help in the prediction of pre-eclampsia in vulnerable patients particularly in resource poor set-ups.

\section{MATERIALS AND METHODS}

This cross-sectional study was carried out on 71 pregnant ladies attending the antenatal clinic in the Gynaecology and Obstetrics Department at College of Medicine and Sagore Dutta Hospital, a tertiary care hospital in Kolkata. All antenatal patients who gave informed consent for the study were included. Pregnant mothers suffering from diabetes, primary renal disease and other medical disorders complicating pregnancy like SLE, heart disease, hypothyroidism, Cushing's, etc. were excluded from the study. Antenatal mothers with multiple pregnancies were also excluded. Fresh, clean voided, midstream urine specimen was collected from the patients for testing for proteinuria. The estimation of protein in the spot urine thus collected was done by the Microprotein kit (pyrogallol-red method) supplied by Coral Clinical Systems on ERBA Chem 5x semiauto analyser. In an acidic medium, proteins combine with Pyrogallol-red and molybdate to form a blue purple coloured complex. The intensity of the colour of this complex as measured at $600 \mathrm{~nm}$ is directly proportional to the amount of proteins present in the sample. This method could be easily adapted to automation as the dye present in the reagent does not stain the walls of the cuvette and thus do not interfere with the measurements.

The statistical data thus compiled was analysed statistically by the IBM SPSS version 16. After calculation of the standard deviation, the data was subjected to tests of normality of Kolmogorov-Smirnov. As per the results obtained, the coverage interval was calculated by the software and reported with the help of a table as described by IUPAC.

\section{RESULTS}

The results obtained after statistical analysis are described below.

\begin{tabular}{|c|c|}
\hline Number of Patients & 48 \\
\hline Mean of All Values & $27.82 \mathrm{mg} / \mathrm{dL}$ \\
\hline Median of All Values & $21.67 \mathrm{mg} / \mathrm{dL}$ \\
\hline Mode (only smallest value is shown) & $10.73 \mathrm{mg} / \mathrm{dL}$ \\
\hline Standard Deviation & 18.02 \\
\hline Standard Error of Mean & 2.71 \\
\hline \multicolumn{2}{|c|}{ Test for Normal Distribution } \\
\hline Kolmogorov-Smirnov test & $P 0.047$ \\
\hline \multicolumn{2}{|c|}{ (The Distribution is not in Normal Distribution as $P<0.05$ ). } \\
\hline Non-parametric coverage interval & $23.99-29.64 \mathrm{mg} / \mathrm{dL}$ \\
\hline $\begin{array}{l}\text { Table 1. Descriptive Statistics of the } \\
\text { from Pregnant Ladies in Se }\end{array}$ & $\begin{array}{l}\text { rinary Microprotein } \\
\text { d Trimester }\end{array}$ \\
\hline
\end{tabular}

\begin{tabular}{|c|c|c|c|c|}
\hline Source & SS & df & MS & \\
\hline Between Treatments & 117.0361 & 2 & 58.5181 & $\mathrm{~F}=0.15259$ \\
\hline Within Treatments & 25694.4364 & 67 & 383.4991 & \\
\hline Total & 25811.4725 & 69 & & \\
\hline \multicolumn{5}{|c|}{ Table 2. Comparision of the Statistics Of Urinary } \\
Microprotein among the Patients belonging to the Three \\
Trimesters (i.e. patients of first Trimester, Patients of \\
Second Trimester and those of Third Trimester) by ANOVA \\
\hline
\end{tabular}

The f-ratio value is 0.15259 . The $\mathrm{p}$-value is 0.858779 . The result is not significant at $\mathrm{p}<0.05$.

\section{DISCUSSION}

The renal physiology undergoes tremendous changes very early during pregnancy. The size of the kidney increases by $1.5 \mathrm{~cm} .{ }^{[10]}$ With the increase in renal plasma flow, the glomerular filtration rate starts rising and may reach $25 \%$ higher as early as the end of the second week of conception. It escalates to $50 \%$ by the beginning of the second trimester. The pregnancy-induced haemodilution leads to a decrease in plasma protein concentration with a consequent decrease in the plasma oncotic pressure within the glomerulus. The renal plasma flow which increases by as much as $80 \%$ at the end of the second trimester may also contribute to the elevation of GFR in pregnancy.[1] This increased GFR coupled with increased permeability of the basement membrane ultimately leads to increased excretion of protein in urine during pregnancy. This haemodilution and hypervolaemia of gestation eventually reverses by the second week postpartum.[11]

As per IFCC, collection and analysis of at least 120 samples is mandatory for establishment of reference interval which may not be always economically viable under strict inclusion and exclusion criteria. This is particularly relevant in the establishment of reference values for those parameters in which the reference method is also costly (e.g. estimation of anti-epileptic drugs, toxic metals, antioxidants, etc.). The distribution of most biological parameters are not Gaussian. Recently, the term 'coverage interval' is preferred over the term 'reference interval' by the International Union of Physical and Applied Chemistry (IUPAC) as well as other leading expert bodies like the International Organisation for Standardisation (ISO). The term coverage interval enjoys a number of advantages. The coverage interval envisages the probability of distribution of the values in the reference population with a specified degree of confidence. Additionally, the degree of uncertainty associated with the value is statistically well-defined.[12] In other words, the term coverage interval is somewhat similar to prediction interval. This uncertainty $(\delta)$, which is a measure of the authenticity of the calculated coverage interval, is independent of the standard deviation. In fact, it is influenced by the level of expectation $(\beta)$, level of confidence $(\gamma)$ and the number of values (n). For this purpose, we undertook to establish the 0.95 coverage interval of proteinuria in pregnancy (level of expectation or $\beta$ ) with a confidence of 0.90, (level of confidence, $\gamma$ ) with a statistically defined level of uncertainty $(\delta)$.

When the sample size for the calculation of the coverage interval is small, it is not possible to define a $95 \%$ confidence interval. In such cases, $90 \%$ confidence interval may be calculated as per the recommendations in the Table 1 of the IUPAC document.[12] Therefore, a fewer number of reference values can be used to estimate a clinically useful interval which is also endowed with defined uncertainty and precision. The coverage interval, similar to the reference interval can be calculated both parametrically as well as nonparametrically. In clinical medicine, any value outside the coverage interval indicates that the individual may be at a greater health risk. Coverage intervals are extremely useful for calculation of toxic doses (the upper limits) of drugs and poison. Smaller coverage uncertainty provides with a higher precision in the estimation of the coverage interval. 
Though the urinary dipstick method is simple and inexpensive to carry out, its major drawback is the high number of false positives, inaccuracy and decreased sensitivity. The term 'trace proteinuria' obtained in the routine dipstick screening method as defined by " +1 " is widely accepted as a marker for pregnancy proteinuria. But this result may be unreliable from the clinical point of view as it largely depends on the maternal hydration status. False positives are also encountered with haematuria, alkaline $\mathrm{pH}$, interference by detergents, drugs, high specific gravity, etc.[13,14] $\mathrm{A}$ large number of false positives would eventually drain the available clinical resources for the management of pre-eclampsia in the name of prevention. In order to avoid these drawbacks, we went ahead with the pyrogallol-red method. Originally described by Fujita,[15] the pyrogallol-red method is one of the few colorimetric methods whose reagents do not adsorb to the walls of the cuvettes and so do not interfere with the measurements. For this reason, this method could be adapted to automated analysers.[16]

The reaction is complete within 5 minutes and the absorbance of the reaction mixture remains unchanged at $25^{\circ} \mathrm{C}-37^{\circ} \mathrm{C}$. So, this method may be easily carried out at peripheral health care centres which have no access to air conditioning as prevalent in this country. The method enjoys a high linearity, almost up to $4000 \mathrm{mg} / \mathrm{dL}$ and has a high sensitivity procedure as well. This method has adequate reproducibility and recovery studies necessary for clinical use. In this study, we used a semi-auto analyser which is relatively inexpensive and may be available in resource poor set-ups like block primary health centres.

In our study, the coverage interval of urinary microprotein in the second trimester is $23.99-29.64 \mathrm{mg} / \mathrm{dL}$ and the mean value of urinary microprotein in the second trimester was $27.82 \mathrm{mg} / \mathrm{dL}$. Comparison of the mean of urinary microprotein in all the three trimesters by ANOVA (vide Table 2 ), showed that there is no statistically significant difference in the mean values of urinary microprotein in the second trimester compared to the first and third. So, for all practical purposes, the coverage interval of urinary microprotein in the second trimester may be applied to the antenatal mothers in the first and third trimesters as well. Usually, a higher number of antenatal mothers seek medical advice in the second trimester (first contact) compared to the first trimester in this part of the country.

Keeping all this in mind, the 0.95 coverage interval of urinary microprotein in pregnancy, with a confidence of 0.90 is calculated to be $23.99-29.64 \mathrm{mg} / \mathrm{dL}$ with an uncertainty of 0.049 as deduced from the table recommended by the IUPAC. In other words, 'the interval [23.99-29.64] will on an average cover $95 \%$ of the distribution of all the values for pregnancy proteinuria such that the probability is at least 0.90 that the interval covers between 0.95-0.049 and $0.95+0.049$ of the distribution.

\section{CONCLUSION}

In this study, we tried to estimate the coverage interval of urinary microprotein in pregnant women attending a tertiary care hospital in Eastern India by the pyrogallol-red method. This is a simple method with adequate linearity and reproducibility which can be easily adapted to peripheral health care establishments with limited infrastructure in rural areas in this country. The IUPAC, ISO and other leading bodies have advocated the usage of the term coverage interval instead of reference interval. The coverage interval is usually declared with the level of confidence, level of expectation, and a defined degree of uncertainty, all of which are taken into account in its calculation in accordance with the recommendations of IUPAC. In this study, we conclude that the coverage interval of urinary microprotein for pregnancy proteinuria is $23.99-29.64 \mathrm{mg} / \mathrm{dL}$ in the population attending a tertiary care hospital in Eastern India as determined by the pyrogallol-red method.

\section{REFERENCES}

[1] Cornelis T, Odutayo A, Keunen J, et al. The kidney in normal pregnancy and pre-eclampsia. Seminars in Nephrology 2011;31(1):4-14.

[2] Khan KS, Wojdyla D, Say L, et al. WHO analysis of causes of maternal death: a systematic review. Lancet 2006;367(9516):1066-74.

[3] Roberts M, Lindheimer MD, Davison JM. Altered glomerular permselectivity to neutral dextrans and heteroporous membrane modeling in human pregnancy. Am J Physiol 1996;270(2 Pt 2):F338-F43.

[4] Higby K, Suiter CR, Phelps JY, et al. Normal values of urinary albumin and total protein excretion during pregnancy. Am J Obstet Gynecol 1994;171(4):984-9.

[5] Price CP, Newall RG, Boyd JC. Use of protein: creatinine ratio measurements on random urine samples for prediction of significant proteinuria: a systematic review. Clinical Chemistry 2005;51(9):1577-86.

[6] Waugh JJ, Clark TJ, Divakaran TG, et al. Accuracy of urinalysis dipstick techniques in predicting significant proteinuria in pregnancy. Obstet Gynecol 2004;103(4):769-77.

[7] Amin SA, Illipilla S, Hebbar S, et al. Quantifying proteinuria in hypertensive disorders of pregnancy. International Journal of Hypertension Article ID 941408, 2014;2014: p. 10.

[8] Waugh JJS, Bell SC, Kilby MD, et al. Optimal bedside urinalysis for the detection of proteinuria in hypertensive pregnancy: a study of diagnostic accuracy. BJOG 2005;112(4):412-7.

[9] Dwyer B, Gorman M, Carroll IR, et al. Urinalysis vs urine protein-creatinine ratio to predict significant proteinuria in pregnancy. Journal of Perinatology 2008;28(7):461-7.

[10] Studd J. The origin and effects of proteinuria in pregnancy. BJOG 1973;80(10):872-83.

[11] Hladunewich MA, Lafayette RA, Derby GC, et al. The dynamics of glomerular filtration in the puerperium. American Journal of Physiology Renal Physiology 2004;286(3):F496-503.

[12] Poulsen OM, Holst E, Christensen JM. Calculation and application of coverage intervals for biological reference values (Technical Report) Pure \& Appl Chem 1997;69(7):1601-11.

[13] Waugh J, Bell SC, Kilby M, et al. Effect of concentration and biochemical assay on the accuracy of urine dipsticks in hypertensive pregnancies. Hypertension in Pregnancy 2001;20(2):205-17. 
[14] Phelan LK, Brown MA, Davis GK, et al. A prospective study of the impact of automated dipstick urinalysis on the diagnosis of pre-eclampsia. Hypertension in Pregnancy 2004;23(2):135-42.

[15] Fujita Y, Mori I, Kitano S. Colour reaction between pyrogallol-red molybdenum (VI) complex and protein. Bunseki Kagaku 1983;32:E379-86.
[16] Watanabe, Kamei S, Ohkubo A, et al. Urinary protein as measured with a pyrogallol-red-molybdate complex, manually and in a Hitachi 726 automated analyzer. Clin Chem 1986;32(8):1551-4. 